\title{
Epidemiology of Frequent Visits to the Emergency Department at a Tertiary Care Hospital in Saudi Arabia: Rate, Visitors' Characteristics, and Associated Factors
}

This article was published in the following Dove Press journal:

International Journal of General Medicine

\author{
Khaled Al-Surimi (iD) ${ }^{1-3}$ \\ Nagarajkumar Yenugadhati $\mathbb{D}^{1,2}$ \\ Naila Shaheen (iD) 2,4 \\ Majed Althagafi ${ }^{1,2,5}$ \\ Majid Alsalamah iD 1,2,5 \\ 'College of Public Health and Health \\ Informatics, King Saud Bin Abdulaziz \\ University for Health Sciences, Riyadh, \\ Kingdom of Saudi Arabia; ${ }^{2}$ King Abdullah \\ International Medical Research Center, \\ Riyadh, Kingdom of Saudi Arabia; ${ }^{3}$ Faculty \\ of Medicine and Health Sciences, Thamar \\ University, Dhamar, Yemen; ${ }^{4}$ King Saud \\ Bin Abdulaziz University for Health \\ Sciences, Riyadh, Kingdom of Saudi \\ Arabia; ${ }^{5}$ Department of Emergency \\ Medicine, College of Medicine, King Saud \\ Bin Abdulaziz University for Health \\ Sciences, Riyadh, Kingdom of Saudi \\ Arabia
}

Background: Emergency department overcrowding is becoming a challenge for the healthcare management system globally and locally. This study aimed to estimate the frequency of ED visits, describe the patients' profile along with visit-related characteristics, and associated factors in a tertiary care hospital.

Methods: A retrospective cohort study was conducted at a tertiary care hospital. The study included patients age 14 years and above visiting the main emergency department in year 2013. Data were extracted from electronic medical records by a qualified data extraction team. Statistical analyses were performed, including the odds ratio and $95 \%$ confidence interval for the factors associated with highly frequent ( $\geq 14$ visits) ED visits using logistic regression models.

Results: There were 150,727 visits to the emergency department within a year. The number of frequent visitors was 7696 (9.38\%), with 42,226 visits (28.01\% of total ED visits). Highly frequent visitors totaled $249(0.30 \%)$, with 5173 visits $(3.43 \%$ of total ED visits). The frequent visitors' average age was 42.55 (SD 20.14), and 48.99 (SD 21.33) for the highly frequent visitors' group. More than half of the emergency visitors were females. The most common complaints among the highly frequent visitors were Gastrointestinal $(21.34 \%)$, followed by Respiratory (13.47\%), Orthopedic (12.57\%), and Cardiovascular (12.43\%). Multivariate analysis indicated that age, history of diabetes, history of cardiac diseases, insurance status, and nationality were significant predicators of highly frequent visits to the hospital emergency.

Conclusion: Frequent and highly frequent visitors to emergency departments represent a significant proportion of adult patients presenting to ED. Their visits constitute almost onethird of total ED visits. Several factors associated with highly frequent ED visits have been identified. This study provides local empirical evidence to develop improvement policy and actions related to chronic issue of frequent and highly frequent visitation to hospital ED.

Keywords: emergency department, frequent visits, hospital, quality improvement, Saudi Arabia

\section{Introduction}

The role of Emergency Department (ED) has changed over time from providing rooms for the resuscitation of severely ill or injured patients to becoming a complex healthcare service setting, using sophisticated technology to provide healthcare that was once given in hospital wards. It has been reported that the ED utilization is
Correspondence: Naila Shaheen Department of Biostatistics and International Medical Research Center, King Saud Bin Abdulaziz University for Health Sciences, Ministry of National Guard-Health Affairs, P.O. Box 22490 Mail Code 1515, Riyadh, II426, Kingdom of Saudi Arabia

Tel +966- I I-4294472

Fax +966-II-4294466

Email drnaila@hotmail.com 
higher than other healthcare settings. In the United States, for example, $11 \%$ of the outpatient services are provided in the ED and almost half of the hospital admissions go through the ED.

Several studies have reported dramatic increase in ED visits. ${ }^{1-3}$ This increased emergency department utilization accounted for about $25 \%$ of visits by less than $10 \%$ of the population. ${ }^{4}$ Studies have indicated that most frequent visitors are patients who consult primary health care physicians, have insurance, and use inpatient and outpatient health care services. ${ }^{5-7}$ A recent study in 2019, has reported that frequent users were $4-6 \%$ of the total ED users but have accounted for $14-47 \%$ of ED visits. ${ }^{8}$ Other studies have reported that the complaints and conditions of frequent ED visitors are not manageable in outpatient and primary care settings. $^{9-11}$

While there is no consensus in literature on the definition of high frequent visits to $\mathrm{ED}$, the most common standard set for frequent ED visiting is four or more visits per year. ${ }^{4,12,13}$ Studies have shown that visiting frequency is highest at $1-3$ visits per year but falls from four visits per year and above. ${ }^{4,12,13}$ The frequency range suggests a visiting pattern that is not coincidental but rather systematic. This has been shown by statistical models comparing multiple cutoffs to determine the diversion between what is expected to happen by chance and what has been observed in reality. ${ }^{12}$ Other studies have attempted to divide frequent visits into subgroups that share typical patient or visit characteristics. For example, a frequent ED visitor with 4-17 visits was found to have higher mortality, more admissions; and is unlikely to leave without being seen. ED visitors of more than 17 or 20 visits are less sick, unlikely to be admitted, and frequently leave ED without being seen. ${ }^{14,15}$ The high frequent visit rates used to be defined as ED visitors with 10, 12, 17, and 20 times per year. ${ }^{16-20}$

In the Middle East context, including the Arabian Gulf States, the reported literature on emergency department frequent visitors and their characteristics, along with the determinant factors, are scarce. In Saudi Arabia, few studies have reported frequent visits to hospital emergency. ${ }^{1,7}$

Local empirical evidence can inform healthcare policymakers, health planners, hospital administrators, and ED heads on implementing improvement initiatives, optimizing utilization of hospital emergency services, and securing high quality and safety for urgent emergency patients. This study aimed primarily to estimate the magnitude of frequent visitors to a hospital emergency department, describe the characteristics of patients and their visits, and identify the associated factors with highly frequent visitation.

\section{Methods}

\section{Study Setting and Design}

This is a retrospective cohort study based on the data extracted from an electronic database at a tertiary care hospital, Riyadh, Saudi Arabia. The emergency department at King Abdulaziz Medical City (KAMC) accommodates 150 beds and manages both pediatric and adult patients, with about $150,000-160,000$ visits per year. KAMC is an academic tertiary center, with a capacity of 1500 beds.

\section{Study Population}

Study participants were adult patients visiting the main emergency department over a one-year period in 2013. An adult patient was defined as greater than or equal to 14 years of age. The 14 years of age cut-off was selected based on local practice in most hospitals. Adults presenting to ED within 24 hours with a chief complaint were included.

\section{Frequent ED Visitor}

A frequent ED visitor refers to a patient who visits the ED four times or more within a year. This definition is considered the most commonly reported description in the literature. ${ }^{4,12,13}$ The ED visit frequency was measured by a single patient visit during the study period from 1 January to 31 December 2013. The total number of ED visits for each patient was counted for 2013. This threshold, although defined as a homogeneous group of occasional visitors to the emergency department, has failed to describe the heterogeneous group of ED frequent visitors. Therefore, we have divided frequent visitors into three groups (less frequent, frequent and highly frequent users) using a data driven approach (see statistical analysis section).

\section{Data Collection}

The data were obtained from the electronic medical records for all the ED visits from 1 January to 31 December 2013, and recorded in an Excel sheet. The collected variables included patients' demographic and clinical characteristics (age, sex, nationality, payment method, medical history, whether a hospital employee or 
not, and co-morbidities), ED visitation data, ie how many times the patient had visited the ED; time of visit; mode of arrival (private car or ambulance), triage and acuity level, length of stay in ED, and the outcome of the visit (admission/discharge home). The eight-hour shift schedule was divided into morning, evening, and night. The insurance status was described as either eligible or ineligible for treatment in tertiary care. The length of ED stay was calculated as the time from ED presentation to the time the electronic health system shown either an order of discharge or admission. It is worth noting that the length of stay does not denote the physical presence in the emergency department. In addition, the history of chronic medical conditions was recorded as a dichotomous (yes/no) variable. Patient acuity was determined by the Canadian Triage and Acuity Scale (CTAS). ${ }^{21}$

The study was approved by King Abdullah International Medical Research Center, Institutional Review Board (IRB) (RC12/156). Data confidentiality was preserved, and no personal identifiers were captured. The study was conducted in accordance with the Declaration of Helsinki. The patient informed consent was not required, since the collected data was deidentified.

\section{Statistical Analysis}

The patient and visit characteristics were reported as frequency and percentages. For the comparison of the categorical variables with respect to ED visit frequency categories, Chi-square and Fisher exact tests were employed. The mean and standard deviation were reported for continuous variables, and the differences among frequent visit groups were evaluated using Kruskal-Wallis test. To determine the breakpoints used to define the frequent and highly frequent ED users, a breakpoint strategy, proposed by Doupe et al was implemented. ${ }^{14}$ Briefly, patient characteristics (as percentages) were plotted against the frequency of ED use, and the breakpoints were determined by the deviations observed in the trend. To identify the determinants of frequent ED users, we used univariate and multivariate logistic regression analyses to examine the association between various patient characteristics and frequent ED users. Two separate logistic regression analyses were performed to compare high-frequency users with frequent users, and high-frequency users with less frequent users. Both unadjusted odds ratios (OR), as well as adjusted odds ratios (AOR) along with their 95\% confidence intervals $(95 \% \mathrm{CI})$ obtained from logistic regression analyses for all the covariates, have been reported. It should be noted that the admission status variable was excluded from logistic regression models due to data unreliability and the unlikelihood of the variable being a predictor of frequent or highly frequent ED use. The discriminatory power and calibration of the multivariate models were evaluated using concordance (C) statistic and Hosmer and Lemeshow (HL) goodness of fit test respectively. All the analyses were performed using SAS software, version 9.4.

\section{Results}

\section{Breakpoints of Total ED Adult Patients Visits by Sex, Age, and Co-Morbidities}

Based on selected patient characteristics, including female patients, patients aged 65 years or more, patients with a history of cardiac disease, hypertension, diabetes, and employment in the hospital, the breakpoints for frequent and highly frequent ED visitors were determined. The breakpoint for frequent ED visits by female patients was determined to be four or more visits based on the deviation in trend observed in the majority of patient characteristics (see Figure 1). For example, the percentage of hospital employees making four visits declined in contrast to higher percentages observed up to three visits. Conversely, a higher rate of patients aged 65 or more visited the ED four times. A similar deviation in trend was detected in patients with a history of diabetes, and a proportion of female patients. Following a similar strategy, the breakpoint for high-frequency visits was determined to be 14 , owing to a drastic decline in the percentage of patients with a history of cardiac disease, hypertension, or diabetes, and patients aged 65 years or more. In addition, a substantially higher proportion of hospital employees visited the ED 14 times. Patients were classified into three groups of ED users based on visit frequency, including less frequent users (1-3 visits), frequent users (4-13 visits), and highly frequent users (14 or more visits).

\section{Rate of ED Visitation and Cohort Characteristics}

A total of 82,046 patients made 150,727 visits during the year 2013 (see Figure 2). Most patients visited the emergency department only once, 51,595 (63\%), which accounted for $34 \%$ of patients' total visits during the study period. The 


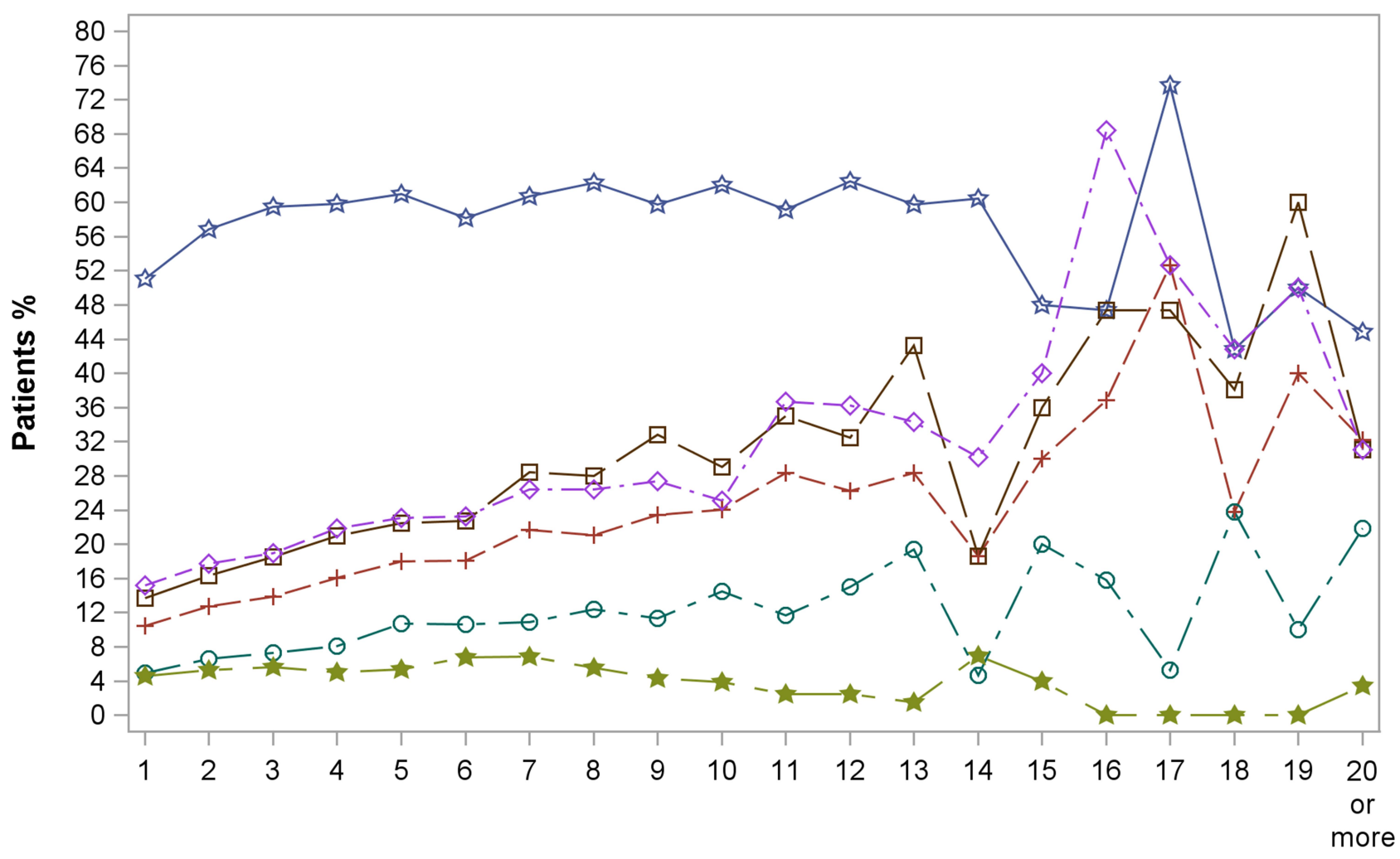

Total Visits To Emergency Department In An Year

\begin{tabular}{|c|c|}
\hline $\begin{array}{c}-\hat{A} \text { Female Patients } \\
-\Theta-\text { History of Cardiac Disease } \\
-\diamond-\text { History of Diabetes }\end{array}$ & $\begin{array}{c}-+- \text { Age }>65 \text { Years } \\
\square-\text { History of Hypertension } \\
\square-\quad \text { Hospital Employee }\end{array}$ \\
\hline
\end{tabular}

Figure I Breakpoints of total ED adult patients' visits by sex, age, and co-morbidities.

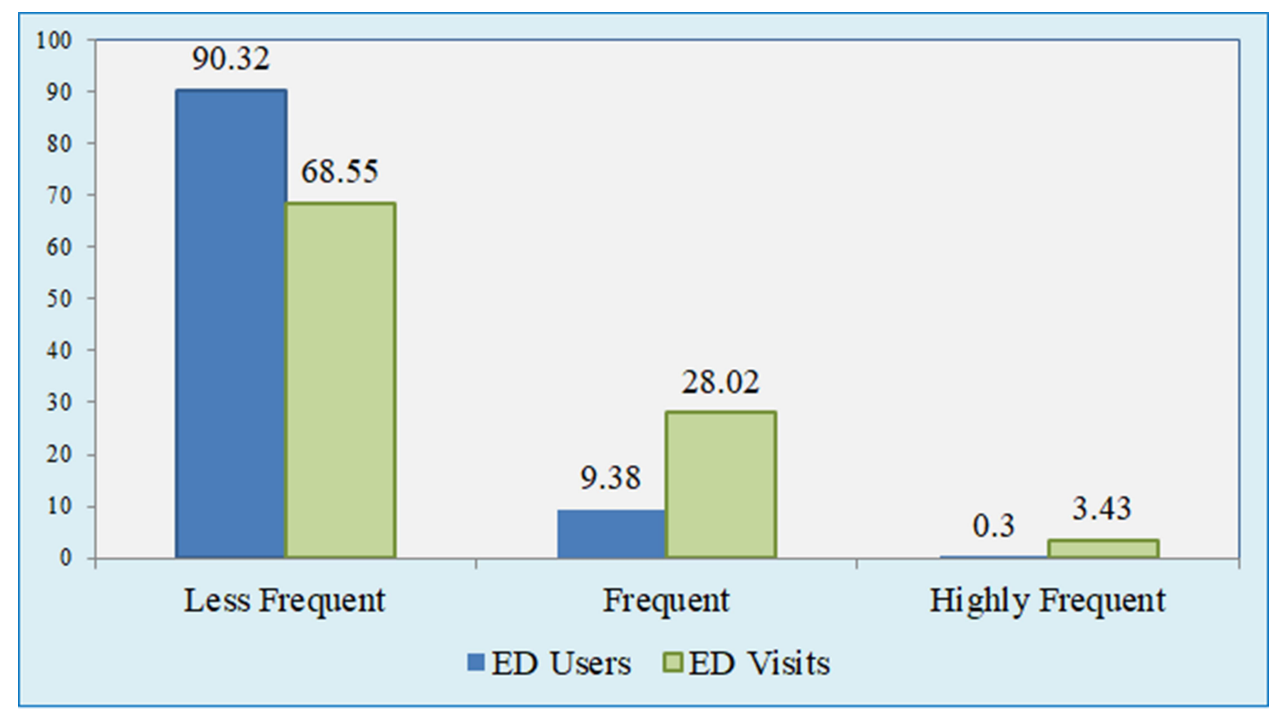

Figure 2 Distribution of total emergency department (ED) users and visits; the total number of ED users $n=82,028$, the total number of $E D$ visits $n=I 50,727$.

majority had less frequent ED visits, 74,101 (90.3\%); 7696 (9.9\%) had frequent ED visits; only $249(0.3 \%)$ had highly frequent visits. The average age was significantly different across the groups, with higher age in the highly frequent visit group ( $\mathrm{p}=<0.0001$ ). Participants were predominantly females 44,058 (53.7\%), Saudi nationals 76,051 (92.7\%), aged 25-44 
Table I Emergency Department Patients' Characteristics by Visits Frequency Types

\begin{tabular}{|c|c|c|c|c|}
\hline \multirow[t]{2}{*}{ Characteristics } & $\begin{array}{l}\text { Less Frequent ED Visitors } \\
\text { (I-3 Visits) }\end{array}$ & $\begin{array}{l}\text { Frequent ED Visitors (4- } \\
\text { I3 Visits) }\end{array}$ & $\begin{array}{l}\text { Highly Frequent ED Visitors } \\
\qquad(\geq 14 \text { Visits) }\end{array}$ & \multirow[t]{2}{*}{ P-value } \\
\hline & $n=74,101(90.32 \%)$ & $n=7696(9.38 \%)$ & $n=249(0.30 \%)$ & \\
\hline Age in years (mean $\pm S D)$ & $38.6 \pm 17.9$ & $42.03 \pm 19.95$ & $49.98 \pm 21.53$ & $<0.0001 *$ \\
\hline \multicolumn{4}{|l|}{ Gender n(\%) } & \multirow{3}{*}{$<0.0001$} \\
\hline Male & $34,796(46.96)$ & $3069(39.8)$ & $123(50.6)$ & \\
\hline Female & $39,305(53.04)$ & $4627(60.12)$ & $126(49.4)$ & \\
\hline \multicolumn{4}{|l|}{ Age in groups $\mathrm{n}(\%)$} & \multirow{5}{*}{$<0.0001$} \\
\hline 14-24 years & $17,722(23.92)$ & $1525(19.82)$ & $25(10.04)$ & \\
\hline $25-44$ years & $32,87 \mid(44.36)$ & $3248(42.2)$ & $90(36.14)$ & \\
\hline $45-64$ years & $15,157(20.45)$ & $1517(19.71)$ & $57(22.89)$ & \\
\hline 65 years or more & $8351(11.27)$ & |406(|8.27) & $77(30.92)$ & \\
\hline \multicolumn{4}{|l|}{ Nationality n(\%) } & \multirow{3}{*}{$<0.0001$} \\
\hline Saudi & $68,452(92.38)$ & $7357(95.6)$ & $242(97.19)$ & \\
\hline Non-Saudi & $5649(7.62)$ & $339(4.4)$ & $7(2.8 \mathrm{I})$ & \\
\hline $\begin{array}{l}\text { History of cardiac disease } \\
\text { (yes) } n(\%)\end{array}$ & $4083(5.5 I)$ & $764(9.93)$ & $4 I(16.47)$ & $<0.0001$ \\
\hline $\begin{array}{l}\text { History of hypertension } \\
\text { (yes) } n(\%)\end{array}$ & $10,917(14.73)$ & $1813(23.56)$ & $85(34.14)$ & $<0.0001$ \\
\hline $\begin{array}{l}\text { History of diabetes (yes) } \\
n(\%)\end{array}$ & $11,910(16.07)$ & $1819(23.64)$ & $97(38.96)$ & $<0.0001$ \\
\hline \multicolumn{2}{|l|}{ Insurance status n(\%) } & & & \multirow{3}{*}{$<0.0001$} \\
\hline Insured fully & $58,418(78.84)$ & $688 I(89.4 I)$ & $210(84.34)$ & \\
\hline Partial insurance & $15,683(21.16)$ & $815(10.59)$ & $39(15.66)$ & \\
\hline \multicolumn{2}{|l|}{ Military status n(\%) } & & & \multirow{3}{*}{0.027} \\
\hline Soldier & $15,294(20.64)$ & $1549(20.13)$ & $67(26.91)$ & \\
\hline Not a soldier & $58,807(79.39)$ & $6147(79.87)$ & 182(73.09) & \\
\hline $\begin{array}{l}\text { Hospital employee (yes) } \\
n(\%)\end{array}$ & $3597(4.85)$ & $4 \mathrm{II}(5.34)$ & $8(3.21)$ & 0.079 \\
\hline \multicolumn{4}{|l|}{$\begin{array}{l}\text { Number of admissions } \\
n(\%)\end{array}$} & \multirow{4}{*}{$<0.0001$} \\
\hline Not admitted & $58,715(79.24)$ & $4154(53.98)$ & $96(38.55)$ & \\
\hline Once & $13,432(18.13)$ & $1910(24.82)$ & $4 I(16.47)$ & \\
\hline 2 or more times & $1954(2.64)$ & $1632(21.21)$ & $112(44.98)$ & \\
\hline
\end{tabular}

Note: *P-value is based on Kruskal-Wallis test, whereas rest of the p-values are based on Chi-square test.

Abbreviations: SD, standard deviation; $n$ (\%), number of subjects (percentage).

years 36,209 (44\%), and insured fully 65,509 (79.8\%). The history of cardiac disease, hypertension, and diabetes was significantly different between groups with more than expected patients in the highly frequent visit category $(p=<0.0001)$. The bulk of patients in the highly frequent visit group (45\%) got admitted to the ward more than once, unlike most frequent (54\%) and less frequent (79\%) visitors who were not admitted (Table 1).

\section{Emergency Department Visit}

\section{Characteristics}

Table 2 reports the characteristics of visits made by visit group. It is worth noting that $9.4 \%$ of frequent users contributed to $28 \%$ of the visits, and a small proportion of highly frequent visitors $(0.3 \%)$ clocked $(3.4 \%)$ of the total visits. Most ED visitors had less urgent and nonurgent cases. However, the proportion of urgent cases 
Table 2 Emergency Department Visit Characteristics by Visits Frequency Types

\begin{tabular}{|c|c|c|c|c|}
\hline Characteristics & $\begin{array}{l}\text { Less Frequent ED } \\
\text { Visitors (I-3 Visits) }\end{array}$ & $\begin{array}{l}\text { Frequent ED Visitors } \\
\text { (4-13 Visits) }\end{array}$ & $\begin{array}{l}\text { Highly Frequent ED } \\
\text { Visitors ( } \geq 14 \text { Visits) }\end{array}$ & P-value \\
\hline & $n=103,328(68.55 \%)$ & $n=42,226(28.01 \%)$ & $n=5$ I $73(3.43 \%)$ & \\
\hline $\begin{array}{l}\text { Duration of stay in ER (Mean } \pm \\
\text { SD) }\end{array}$ & $4.01 \pm 8.16$ & $4.39 \pm 5.98$ & $4.96 \pm 5.86$ & $<0.000$ \\
\hline $\begin{array}{l}\text { Acuity level } \\
\text { Emergent (Levels I-2) } \\
\text { Urgent (Level 3) } \\
\text { Less Urgent (Level 4) } \\
\text { Non-urgent (Level 5) } \\
\text { Unknown }\end{array}$ & $\begin{array}{c}3648(3.53) \\
29,033(28.1) \\
47,944(46.4) \\
20,165(19.52) \\
2538(2.46)\end{array}$ & $\begin{array}{c}1586(3.76) \\
14,715(34.85) \\
19,087(45.2) \\
6409(15.18) \\
429(1.02)\end{array}$ & $\begin{array}{c}196(3.79) \\
205 \mid(39.65) \\
236 \mid(45.64) \\
521(10.07) \\
44(0.85)\end{array}$ & $<0.0001$ \\
\hline $\begin{array}{l}\text { Presenting complaints } \mathrm{n}(\%) \\
\text { Gastrointestinal } \\
\text { Orthopedic } \\
\text { Obstetrics \& Gynecology } \\
\text { Cardiovascular } \\
\text { Neurologic } \\
\text { ENT } \\
\text { Respiratory } \\
\text { General and minor complaints } \\
\text { Genitourinary } \\
\text { Skin } \\
\text { Unknown } \\
\text { Ophthalmology } \\
\text { Mental health } \\
\text { Environmental health and } \\
\text { Substance misuse }\end{array}$ & $\begin{array}{c}19,743(19.1 I) \\
16,378(15.85) \\
10,244(9.91) \\
9157(8.86) \\
8599(8.32) \\
7659(7.4 I) \\
7783(7.53) \\
8174(7.91) \\
4892(4.73) \\
3559(3.44) \\
2768(2.68) \\
2004(1.94) \\
377(0.36) \\
95(0.09)\end{array}$ & $\begin{array}{c}9204(21.8) \\
5327(\mid 2.62) \\
4782(\mid 1.32) \\
359 \mid(8.5) \\
354 \mid(8.39) \\
2552(6.04) \\
4339(10.28) \\
3958(9.37) \\
2274(5.39) \\
1036(2.45) \\
485(1.15) \\
625(I .48) \\
139(0.33) \\
13(0.03)\end{array}$ & $\begin{array}{c}1104(21.34) \\
650(12.57) \\
243(4.7) \\
643(12.43) \\
507(9.8) \\
233(4.5) \\
697(13.47) \\
519(10.03) \\
320(6.19) \\
129(2.49) \\
33(0.64) \\
39(0.75) \\
30(0.58) \\
2(0.04)\end{array}$ & $<0.0001$ \\
\hline $\begin{array}{l}\text { Radiology requests } \mathrm{n}(\%) \\
\text { No imaging required } \\
\text { Imaging required }\end{array}$ & $\begin{array}{c}96,450(3.34) \\
6878(6.66)\end{array}$ & $\begin{array}{c}38,618(91.46) \\
3608(8.54)\end{array}$ & $\begin{array}{c}4759(92) \\
414(8)\end{array}$ & $<0.0001$ \\
\hline $\begin{array}{l}\text { Medication requested } \mathrm{n}(\%) \\
\text { No prescription required } \\
\text { Prescription given }\end{array}$ & $\begin{array}{l}91,276(88.34) \\
12,052(11.66)\end{array}$ & $\begin{array}{c}36,789(87.12) \\
5437(12.88)\end{array}$ & $\begin{array}{c}4606(89.04) \\
567(10.96)\end{array}$ & $<0.0001$ \\
\hline $\begin{array}{l}\text { Emergency visit time } \mathrm{n}(\%) \\
\text { Morning shift } \\
\text { Evening shift } \\
\text { Night shift }\end{array}$ & $\begin{array}{l}40,706(39.39) \\
41,600(40.26) \\
21,022(20.34)\end{array}$ & $\begin{array}{l}15,855(37.55) \\
16,879(39.97) \\
9492(22.48)\end{array}$ & $\begin{array}{c}2030(39.24) \\
2062(39.86) \\
1081(20.9)\end{array}$ & $<0.0001$ \\
\hline $\begin{array}{l}\text { Day of the week } \mathrm{n}(\%) \\
\text { Sunday } \\
\text { Monday } \\
\text { Tuesday } \\
\text { Wednesday } \\
\text { Thursday } \\
\text { Friday } \\
\text { Saturday }\end{array}$ & $\begin{array}{l}15,639(15.14) \\
14,777(\mid 4.3) \\
15,083(14.6) \\
14,019(13.57) \\
13,966(\mid 3.52) \\
14,109(13.65) \\
15,735(\mid 5.23)\end{array}$ & $\begin{array}{l}6286(14.89) \\
6110(14.47) \\
6058(14.35) \\
5715(13.52) \\
5758(13.64) \\
5910(14) \\
6389(15.13)\end{array}$ & $\begin{array}{l}727(\mid 4.05) \\
732(\mid 4.15) \\
746(\mid 14.42) \\
738(\mid 4.27) \\
762(14.73) \\
67 \mid(\mid 2.97) \\
797(|5.4|)\end{array}$ & 0.086 \\
\hline
\end{tabular}

(Continued) 
Table 2 (Continued).

\begin{tabular}{|c|c|c|c|c|}
\hline \multirow[t]{2}{*}{ Characteristics } & $\begin{array}{l}\text { Less Frequent ED } \\
\text { Visitors ( } 1-3 \text { Visits) }\end{array}$ & $\begin{array}{l}\text { Frequent ED Visitors } \\
\qquad(4-13 \text { Visits) }\end{array}$ & $\begin{array}{l}\text { Highly Frequent ED } \\
\text { Visitors ( } \geq 14 \text { Visits) }\end{array}$ & \multirow[t]{2}{*}{ P-value } \\
\hline & $n=103,328(68.55 \%)$ & $n=42,226(28.0 I \%)$ & $n=5173(3.43 \%)$ & \\
\hline \multicolumn{5}{|c|}{$\begin{array}{l}\text { Duration of stay in emergency } \\
\text { department } n(\%)\end{array}$} \\
\hline I hr. or less & $24,413(23.63)$ & $7227(17.12)$ & $685(I 3.24)$ & \multirow{7}{*}{$<0.0001$} \\
\hline I.0-2.0 hrs. & $21,643(20.95)$ & $7943(18.81)$ & $879(16.99)$ & \\
\hline $2.0-3.5 \mathrm{hrs}$. & $22,106(21.39)$ & $8906(21.09)$ & $1049(20.28)$ & \\
\hline $3.5-6.0 \mathrm{hrs}$ & $17,964(17.39)$ & $8655(20.5)$ & $1128(21.81)$ & \\
\hline $6.0-10.0$ hrs. & $10,596(10.25)$ & $6082(14.4)$ & $896(17.32)$ & \\
\hline More than $10 \mathrm{hrs}$. & 6603(6.39) & $34 I I(8.08)$ & $536(10.36)$ & \\
\hline Unknown ${ }^{\dagger}$ & 3 & 2 & 0 & \\
\hline
\end{tabular}

Notes: *P-value is based on Kruskal-Wallis test, whereas rest of the $p$-values are based on Chi-square test; $n(\%)=$ number of visitors $\left(\right.$ percentage); ${ }^{\dagger}$ unknown visits $(n=5)$ were excluded from the analysis.

was higher among frequent $14,715(34.9 \%)$ and highly frequent $2051(39.7 \%)$ visitors compared to less frequent visitors 29,033 (28.1\%). The predominant complaints from less frequent users were gastrointestinal, orthopedic, obstetrics and gynecology, and cardiovascular. Among frequent users, the chief complaints were similar to those of less frequent users, but the fourth major complaint was of respiratory origin. Highly frequent ED users presented with gastrointestinal $(21.3 \%)$, respiratory $(13.5 \%)$, orthopedic $(12.6 \%)$, and cardiovascular (12.4\%) complaints. The median duration of stay in the emergency department before the patient was either admitted or discharged was significantly higher among frequent visitors and the highest among highly frequent visitors $(p<0.0001)$. The ED visitation was not statistically different throughout the days of the week $(p=0.086)$.

\section{Patient Factors Associated with Highly Frequent Visits}

Table 3 shows the OR and AOR with corresponding 95\% $\mathrm{CI}$ for the association between highly frequent ED visitors and various patient characteristics using frequent visitors as a comparison group. The logistic regression models were based on 249 highly frequent ED visitors and 7696 frequent ED visitors. Notably, an increased risk of highly frequent ED use was observed among older age groups (with reference to the 14-24 years group) $(\mathrm{p}<0.05)$. In addition, a 1.5-fold and 1.6-fold increased risk of highly frequent ED use was observed among partially insured patients and patients with a history of diabetes, respectively $(\mathrm{p}<0.05)$. The multivariate model was well-calibrated (HL test $\mathrm{p}=0.7305)$ and exhibited reasonable discriminatory power $(\mathrm{C}$-statistic $=0.6)$. The OR and AOR and their 95\% CI for the association between highly frequent ED use and various patient characteristics using less frequent ED users as a comparison group were obtained through 249 highly frequent ED users and 74,101 less frequent ED users (Table 4). A minimum of 1.5 -fold increased risk of highly frequent ED use was observed among various age groups (with reference to the 14-24 years group), patients with a history of cardiac disease, and patients with a history of diabetes $(p<0.05)$. Further, non-Saudi nationals were less prone to highly frequent ED use than Saudi nationals $(\mathrm{p}<0.05)$. This multivariate model was well-calibrated (HL test $\mathrm{p}=0.622$ ) and exhibited good discriminatory power $(\mathrm{C}$-statistic $=0.7)$.

\section{Discussion}

Emergency department overcrowding is becoming a challenge for the healthcare management system globally ${ }^{22}$ and locally. ${ }^{1}$ In Saudi Arabia, studies about ED visits mainly focus on a specific study population, ie drug-related problems, $^{23}$ triage system issues, ${ }^{24}$ non-urgent visits, ${ }^{25}$ duration of revisit, ${ }^{26}$ or a specific group of patients, ie post bariatric surgery ${ }^{27}$ and sickle-cell disease. ${ }^{28}$ To our knowledge, the current study is among the few targeting all the adult patients frequenting the emergency department in tertiary care in Saudi Arabia.

During the study period, the rate of frequent visitors is evidently trending. It has been found that the most common definition of frequent visitation was four and more 
Table 3 Comparison of Patient's Characteristics Between Frequent and Highly Frequent Visitors in Emergency Department

\begin{tabular}{|c|c|c|c|c|c|c|}
\hline \multirow[t]{2}{*}{ Characteristics } & \multirow{2}{*}{$\begin{array}{l}\text { Frequent Visitors (4-13 } \\
\text { Visits) n=7696 (9.38\%) }\end{array}$} & \multirow{2}{*}{$\begin{array}{c}\text { Highly Frequent Visitors }(\geq 14 \\
\text { Visits) } n=249(0.30 \%)\end{array}$} & \multicolumn{2}{|c|}{ Univariate } & \multicolumn{2}{|c|}{ Multivariate } \\
\hline & & & OR & $95 \% \mathrm{Cl}$ & AOR & $95 \% \mathrm{Cl}$ \\
\hline \multicolumn{7}{|l|}{ Gender } \\
\hline Male & $3069(39.9)$ & $123(50.6)$ & Ref & & Ref & \\
\hline Female & $4627(60.1)$ & $126(49.4)$ & 0.68 & $(0.53-0.88)$ & 0.81 & $(0.57-1.14)$ \\
\hline \multicolumn{7}{|l|}{ Age group } \\
\hline 14-24 years & $1525(19.82)$ & $25(10.04)$ & Ref & & Ref & \\
\hline $25-44$ years & $3248(42.2)$ & $90(36.14)$ & 1.69 & $(1.08-2.64)$ & 1.7 & $(1.06-2.72)$ \\
\hline $45-64$ years & $1517(19.71)$ & $57(22.89)$ & 2.29 & (I.43-3.69) & 1.96 & $(1.16-3.31)$ \\
\hline 65 years or more & $1406(18.27)$ & $77(20.92)$ & 3.34 & $(2.12-5.28)$ & 2.61 & $(1.52-4.46)$ \\
\hline \multicolumn{7}{|l|}{ Nationality } \\
\hline Saudi & $7357(95.6)$ & 242(97.19) & Ref & & Ref & \\
\hline Non-Saudi & $339(4.4)$ & $7(2.81)$ & 0.63 & $(0.29-1.34)$ & 0.68 & $(0.30-1.57)$ \\
\hline \multicolumn{7}{|l|}{$\begin{array}{l}\text { History of cardiac } \\
\text { disease }\end{array}$} \\
\hline Yes & $764(9.93)$ & $4 I(16.47)$ & 1.79 & $(1.27-2.52)$ & 1.08 & $(0.74-1.59)$ \\
\hline No & 6932(90.07) & $208(83.53)$ & Ref & & Ref & \\
\hline \multicolumn{7}{|l|}{$\begin{array}{l}\text { History of } \\
\text { hypertension }\end{array}$} \\
\hline Yes & $1813(23.65)$ & $85(34.14)$ & 1.68 & $(1.29-2.20)$ & 0.83 & $(0.57-1.22)$ \\
\hline No & $5883(76.44)$ & $164(65.86)$ & Ref & & Ref & \\
\hline \multicolumn{7}{|c|}{ History of diabetes } \\
\hline Yes & $1819(23.64)$ & $97(38.96)$ & 2.06 & $(1.59-2.67)$ & 1.6 & $(1.12-2.29)$ \\
\hline No & $5877(76.36)$ & $152(6 \mid .04)$ & Ref & & Ref & \\
\hline \multicolumn{7}{|l|}{ Insurance status } \\
\hline Insured fully & $688 I(89.4 I)$ & $210(84.34)$ & Ref & & Ref & \\
\hline Partial insurance & $815(10.59)$ & $39(15.66)$ & 1.57 & $(1.11-2.22)$ & 1.52 & $(1.05-2.26)$ \\
\hline \multicolumn{7}{|l|}{ Military status } \\
\hline Soldier & $1549(20.13)$ & $67(26.91)$ & 1.46 & $(1.10-1.94)$ & 1.26 & $(0.83-1.90)$ \\
\hline Not a soldier & $6147(79.87)$ & I82(73.09) & Ref & & Ref & \\
\hline \multicolumn{7}{|l|}{ Hospital employee } \\
\hline Yes & $4 \mathrm{II}(5.34)$ & $8(3.21)$ & 0.59 & $(0.29-1.20)$ & 0.85 & $(0.38-1.91)$ \\
\hline No & $7285(94.66)$ & $24 I(96.79)$ & Ref & & Ref & \\
\hline \multicolumn{7}{|c|}{ Number of admissions } \\
\hline Not admitted & $4154(53.98)$ & $96(38.55)$ & Ref & & - & - \\
\hline Once & $1910(24.82)$ & $4 I(16.47)$ & 0.93 & $(0.64-1.33)$ & - & - \\
\hline 2 or more times & $1632(21.21)$ & $112(44.98)$ & 2.97 & $(2.25-3.93)$ & - & - \\
\hline
\end{tabular}

Note: Bold AOR measures indicate statistically significant values at 0.05 significance level.

Abbreviations: $\mathrm{n}$, number of patients; OR, odds ratio; AOR, adjusted odds ratio; $95 \% \mathrm{Cl}, 95 \%$ confidence interval; Ref, reference category.

visits per year. A small proportion of patients that made frequent and highly frequent visits accounted for $31.4 \%$ of the total ED visits. This percentage is higher than what has been reported in the literature, $(8 \%)$ in $2006,{ }^{4}(28 \%)$ in 2013. ${ }^{13}$ The large proportion of visits should prompt administrators and policymakers to examine the characteristics of the disproportionately fewer patients and develop interventions that reduce their visit numbers.${ }^{4,11}$ Moreover, almost half of the patients presented with less urgent conditions, a consistent finding across visit frequency groups. This finding is consistent with what was reported in the literature. ${ }^{5,6,15,29}$ 
Table 4 Comparison of Patient's Characteristics Between Less Frequent and Highly Frequent Visitors in Emergency Department

\begin{tabular}{|c|c|c|c|c|c|c|}
\hline \multirow[t]{2}{*}{ Characteristics } & \multirow{2}{*}{$\begin{array}{l}\text { Less Frequent Visitors } \\
\begin{array}{c}\text { ( }-3 \text { Visits) } n=74,101 \\
(90.32 \%)\end{array}\end{array}$} & \multirow{2}{*}{$\begin{array}{c}\text { Highly Frequent ED } \\
\text { Visitors ( } \geq \text { 14 Visits) } n=249 \\
(0.30 \%)\end{array}$} & \multicolumn{2}{|c|}{ Univariate } & \multicolumn{2}{|c|}{ Multivariate } \\
\hline & & & OR & $95 \% \mathrm{Cl}$ & AOR & $95 \% \mathrm{Cl}$ \\
\hline \multicolumn{7}{|l|}{ Gender } \\
\hline Male & $34,796(47.0)$ & $123(50.6)$ & Ref & & Ref & \\
\hline Female & $39,305(53.0)$ & $126(49.4)$ & 0.9 & $(0.7 I-1.16)$ & 1.02 & $(0.73-1.44)$ \\
\hline \multicolumn{7}{|l|}{ Age group } \\
\hline 14-24 years & $17,722(23.92)$ & $25(10.04)$ & Ref & & Ref & \\
\hline $25-44$ years & $32,87 \mid(44.36)$ & $90(36.14)$ & 1.94 & $(1.25-3.02)$ & 1.82 & (1.14-2.89) \\
\hline $45-64$ years & $15,157(20.45)$ & $57(22.89)$ & 2.67 & $(1.67-4.27)$ & 1.81 & $(1.07-3.05)$ \\
\hline 65 years or more & $8351(11.27)$ & $77(30.82)$ & 6.54 & $(4.16-10.27)$ & 3.35 & $(1.95-5.77)$ \\
\hline \multicolumn{7}{|l|}{ Nationality } \\
\hline Saudi & $68,452(92.38)$ & $242(97.19)$ & Ref & & Ref & \\
\hline Non-Saudi & $5649(7.62)$ & $7(2.8 I)$ & 0.35 & $(0.17-0.74)$ & 0.39 & $(0.17-0.91)$ \\
\hline \multicolumn{7}{|c|}{ History of cardiac disease } \\
\hline Yes & $4083(5.51)$ & $4 I(16.47)$ & 3.38 & $(2.42-4.73)$ & 1.52 & $(1.03-2.23)$ \\
\hline No & $70,018(94.49)$ & $208(83.53)$ & Ref & & Ref & \\
\hline \multicolumn{7}{|c|}{ History of hypertension } \\
\hline Yes & $10,917(14.73)$ & $85(34.14)$ & 3.00 & $(2.3 \mathrm{I}-3.90)$ & 1.22 & $(0.83-1.87)$ \\
\hline No & $63,184(85.27)$ & $164(65.86)$ & Ref & & Ref & \\
\hline \multicolumn{7}{|c|}{ History of diabetes } \\
\hline Yes & $11,910(16.07)$ & $97(38.96)$ & 3.33 & $(2.58-4.30)$ & 1.82 & $(1.27-2.61)$ \\
\hline No & $62,191(83.93)$ & $152(61.04)$ & Ref & & Ref & \\
\hline \multicolumn{7}{|l|}{ Insurance status } \\
\hline Insured fully & $58,418(78.84)$ & $210(84.34)$ & Ref & & Ref & \\
\hline Partial insurance & $15,683(21.16)$ & $39(15.66)$ & 0.69 & $(0.49-0.97)$ & 0.81 & $(0.55-1.19)$ \\
\hline \multicolumn{7}{|l|}{ Military status } \\
\hline Soldier & $15,294(20.64)$ & $67(26.91)$ & 1.42 & $(1.07-1.87)$ & 1.25 & $(0.83-1.87)$ \\
\hline Not a soldier & $58,807(79.36)$ & I82(73.09) & Ref & & Ref & \\
\hline \multicolumn{7}{|l|}{ Hospital employee } \\
\hline Yes & $3597(4.85)$ & $8(3.21)$ & 0.65 & $(0.32-1.32)$ & 1.25 & $(0.56-2.78)$ \\
\hline No & $70,504(95.15)$ & $24 I(96.79)$ & Ref & & Ref & \\
\hline \multicolumn{7}{|c|}{ Number of admissions } \\
\hline Not admitted & $58,715(79.24)$ & $96(38.55)$ & Ref & & - & - \\
\hline Once & $13,432(18.13)$ & $4 I(16.47)$ & 1.87 & $(1.30-2.69)$ & - & - \\
\hline 2 or more times & $1954(2.64)$ & II2(44.98) & 35.06 & $(26.59-46.21)$ & - & - \\
\hline
\end{tabular}

Note: Bold AOR measures indicate statistically significant values at 0.05 significance level.

Abbreviations: $n$, number of patients; OR, odds ratio; $A O R$, adjusted odds ratio; $95 \% \mathrm{Cl}, 95 \%$ confidence interval; Ref, reference category.

The age distribution in the study reflects the population pyramid in Saudi Arabia, with majority of the population being young. The highest visiting age group ranged from 25 to 44 years ( $44 \%$ of patients). However, this finding contrasts with ${ }^{7}$ showing different age groups attending ED both in frequent and non-frequent visits. This discrepancy could be attributed to the nature of this study being a self-reporting survey and sampling of population rather than the entire population attending hospital emergency. ${ }^{30}$ In this study, the ED visits were detected more in the 25-44 years age group, in contrast with 45-64 years as reported by Hooker et al. ${ }^{31}$ Overall, previous studies have shown inconclusive conclusion due to age difference between frequent users and non-frequent users, as the current study also supports. $^{5,20,30,32,33}$ 
A small body of literature has indicated a slight difference in age, apportioning infrequent ED visits to the young groups. ${ }^{5,19,33}$ In the study by Liu et al, ${ }^{32}$ a very young (25 years old) age group was a characteristic of the less frequent visitor. In contrast, the peak age in frequent visiting was found to belong to the middle-aged 25-64 years. ${ }^{9}$ It has been reported that frequent visits to the hospital emergency might be due to the lack of primary care providers or the lack of insurance. However, some studies have challenged the hypothesis and reported that lack of insurance was also prevalent among frequent group. ${ }^{30,33,34}$ Vinton et al (2014) stated that countries with universal insurance support the idea that the lack of insurance is not considered a factor. Likewise, a study in the Veteran Health Administration network in the United States reported that insurance status was not a factor, as authors pointed out that despite the unlikelihood of insurance to be a factor, visit frequency patterns between patients were evident. ${ }^{20}$ On the other hand, some studies have shown a difference in the insurance status between the less frequent and the frequent users of hospital emergency. ${ }^{4,5}$ The current study shows that female patients had more visits (53\%), which supports local studies, (55\%) females reported by Al-Otmy etal; (57\%) by Zakaria etal, and international literature Hooker etal Yet, a study has yielded equal numbers of ED visits by males and females. ${ }^{24}$ In general, studies have reported different distribution of frequent visitation across gender; males dominance was reported in Singapore, ${ }^{9}$ in females ${ }^{31}$ or unrelated to gender. ${ }^{35}$ It has been reported in the literature that when visiting frequency increases, the percentage of males increases. ${ }^{5,14,32}$ In contrast, findings in the Saudi context have shown that males were three times more likely to be in the frequent users' groups than females. ${ }^{7}$ Other studies reported no significant differences in gender between the frequent and none frequent ED visitors. ${ }^{15,30,34-37}$ The acuity level in this study mostly features less urgent cases, which accords with other studies. ${ }^{25}$ The chronic diseases, reported in this study, are lower than that of AlOtmy etal. ${ }^{25}$ One of the possible explanations might be due to that all public hospitals in Saudi Arabia have to accept all patients visiting the ED, which encourage patients to bypass the primary care centers, ignoring nature of ED services that must be for life saving conditions. Previous literature has discussed different reasons for seeking ED visits. In the current study, the five most common reasons for visiting ED were gastrointestinal symptoms (36\%), followed by orthopedic (27.2\%), gynecology (18.6\%), cardiovascular (16.3\%), and neurologic (15.4\%). Similarly, a study has reported abdominal problems as the top most complaints (Hooker etal, 2019). Mental health and substance abuse were also identified as common reasons for ED visits. ${ }^{31}$

The study has shown that being a female is a significant predictor of highly frequent emergency visiting. While this finding is consistent with two previous studies, ${ }^{4,33}$ most studies have concluded that being a male can be a predictor of highly frequent emergency visits. $5,14,17,19,32,36$ Furthermore, one study ${ }^{38}$ has revealed that not only the percentage of males increases with frequency but also their mortality rate.

The most significantly associated health conditions with frequent and highly frequent visits were diabetes, cardiac diseases, and respiratory problems. This finding aligns with what has been reported in the literature by a Saudi community survey. ${ }^{39}$ In this study, an increasing tendency was observed among frequent users to be diabetic, hypertensive, have a medical history, or cardiac disease, which also corroborates earlier findings in the literature. ${ }^{14,19,20,29,33}$ These findings postulate that frequent visitors to ED are medically vulnerable.

Frequent visitors had more admissions into the hospital over the study period. The status of having at least oneadmission rate goes up over time. This aligns with earlier studies. $^{5,7,11,30}$ On the other hand, several studies have revealed initial increase in the admission rates then dropdown rates as the visiting frequency exceeds 17 visits per year, which is not supported by the current study. ${ }^{15,17}$ This discrepancy might reflect the poor health conditions in our population or suggest that local medical practice has a low threshold to admit frequent visiting patients. We think this need to be investigated further in future studies.

As for the outcome of the visit being admitted or discharged, there was no difference between occasional and frequent visitors, which corresponds to previous findings, ${ }^{5}$ except for the highly frequent visitors who exhibit a drop in admission rates per visit. Seven categories of the patient's complaints present the most common complaints that constitute the majority of ED visits. This findings also concurs with many previous findings. $5,6,15,34$ Obstetrics and gynecology-related complaints are more prevalent in occasional visitors compared to highly frequent visitors. Categories of cardiovascular, neurologic, and respiratory showed an increasing percentage that correlates with the visiting frequency. The lack of standardization for presenting complaints categories raised an issue 
when comparing our data to other studies. We used a standardized method by presenting an abbreviated list of complaints, developed by the Canadian Health Information. $^{21,40,41}$ The tendency towards complaints aggravated by cardiovascular and respiratory problems compared to occasional visit complaints has also been demonstrated in the literature. ${ }^{15}$ The visiting time, day, or month has not proven to be a factor for frequent visiting. This finding has also been supported by earlier findings $5,11,29,30,37$ except for a study demonstrating an increased odds of frequent visiting for people attending at night shift. ${ }^{15}$

\section{Strengths and Limitations}

This study explores visit frequency and patient's characteristics in Saudi Arabia, with a large sample size. The study was conducted in a tertiary care hospital with high annual ED visits. Yet, several limitations need to be addressed. First, manual data extraction for medical diagnosis was challenging since the information was not available electronically. Second, the study data were collected from a single hospital. In other words, a multicenter study would have provided more insights. Moreover, the extracted data is limited to one year period. Third, we did not include data about the utilization of other healthcare services like outpatient clinics, dialysis centers, and home healthcare. The utilization of services can unravel a patient's visiting pattern and whether related to dissatisfaction with other healthcare services or actual emergency conditions that cannot be managed in the outpatient setting.

\section{Conclusion}

Frequent visits place a significant burden on the hospital emergency department, accounting for more than one-third of the total ED visits. This mirrors the international data about emergency department frequent visiting. Factors significantly associated with highly frequent ED visits are age group, history of chronic disease, including diabetes and cardiac disease, Saudi nationality, and partial insurance coverage. The most frequent complaints among frequent visitors are related to gastrointestinal, cardiovascular, orthopedic, and respiratory systems. No significant difference was observed between less frequent, frequent, or highly frequent visitors with respect to acuity level and admission to the hospital. This study findings provide empirical evidence that could help in developing policy interventions as well as initiating quality improvement projects addressing the issue of frequent ED visitors. From primary care perspective, sustaining effective coordination between EDs and family physicians and primary care providers consider among the proposed solutions for managing ED crowdedness and frequent users. ${ }^{13}$ Likewise, effective communication between care providers is a key component of a case-management approach, ${ }^{42-44}$ especially for chronic conditions. Further studies are also recommended, in particular a qualitative study, exploring the rationale and cultural factors behind ED frequent visitation.

\section{Implications and Future Research}

Methodological studies can help define the optimum number and threshold for visiting frequency in line with our culture, people, and health infrastructure. Different communities call for a different threshold for frequent visiting. The field of quantitative research could be enriched by qualitative studies addressing the characteristics of specific groups of patients like patient's COPD, asthma, heart failure, connective tissue disease, cancer, sickle-cell disease, among whom are frequent ED visitors. Unfortunately, qualitative studies garner little attention from medical researchers. Incentives must be introduced to foreground the role of qualitative research and integrate research capabilities with family medicine and emergency medicine.

\section{Acknowledgments}

Special thanks go to Ms. Baidaa Alrumaih for her language assistance and copy editing.

\section{Disclosure}

The authors report no conflicts of interest in this work.

\section{References}

1. Abualenain JT. Characteristics of emergency department visits at King Abdulaziz University Hospital: a one year analysis. J King Abdulaziz Univ Med Sci. 2018;25(1):1-7. doi:10.4197/Med.25-1.1

2. Pines JM, Hilton JA, Weber EJ, et al. International perspectives on emergency department crowding. Acad Emerg Med off J Soc Acad Emerg Med. 2011;18(12):1358-1370. doi:10.1111/j.1553-2712.2011. 01235.x

3. Almalki ZS, Albassam AA, Alnakhli MA, Alnusyan MF, Alanazi FN, Alqurashi MS. National rates of emergency department visits associated with diabetes in Saudi Arabia, 2011-2015. Ann Saudi Med. 2019;39(2):71-76. doi:10.5144/0256-4947.2019.71

4. Hunt KA, Weber EJ, Showstack JA, Colby DC, Callaham ML. Characteristics of frequent users of emergency departments. Ann Emerg Med. 2006;48(1):1-8. doi:10.1016/j.annemergmed.2005.12.030

5. Mandelberg JH, Kuhn RE, Kohn MA. Epidemiologic analysis of an urban, public emergency department's frequent users. Acad Emerg Med. 2000;7(6):637-646. doi:10.1111/j.1553-2712.2000.tb02037.x 
6. Byrne M, Murphy AW, Plunkett PK, McGee HM, Murray A, Bury G. Frequent attenders to an emergency department: a study of primary health care use, medical profile, and psychosocial characteristics. Ann Emerg Med. 2003;41(3):309-318. doi:10.1067/ mem.2003.68

7. Alghanim SA, Alomar BA. Frequent use of emergency departments in Saudi public hospitals: implications for primary health care services. Asia Pacific J Public Heal. 2015;27(2):NP2521-NP2530. doi: $10.1177 / 1010539511431603$

8. Giannouchos TV, Kum H-C, Foster MJ, Ohsfeldt RL. Characteristics and predictors of adult frequent emergency department users in the United States: a systematic literature review. J Eval Clin Pract. 2019;25(3):420-433. doi:10.1111/jep.13137

9. Chan BTB, Ovens HJ. Frequent users of emergency departments. Do they also use family physicians' services? Can Fam Physician. 2002;48(10):1654-1660.

10. Cydulka RK. Characteristics of occasional and frequent emergency department users: do insurance coverage and access to care matter? Ann Emerg Med. 2004;44(5):554. doi:10.1016/j.annemergmed.20 04.08.031

11. Dent A, Hunter G, Webster AP. The impact of frequent attenders on a UK emergency department. Eur J Emerg Med. 2010;17(6):332-336. doi:10.1097/MEJ.0b013e328335623d

12. Locker TE, Baston S, Mason SM, Nicholl J. Defining frequent use of an urban emergency department. Emerg Med J. 2007;24(6):398-401. doi:10.1136/emj.2006.043844

13. Minassian A, Vilke GM, Wilson MP. Frequent emergency department visits are more prevalent in psychiatric, alcohol abuse, and dual diagnosis conditions than in chronic viral illnesses such as hepatitis and human immunodeficiency virus. J Emerg Med. 2013;45(4):520525. doi:10.1016/j.jemermed.2013.05.007

14. Doupe MB, Palatnick W, Day S, et al. Frequent users of emergency departments: developing standard definitions and defining prominent risk factors. Ann Emerg Med. 2012;60(1):24-32. doi:10.1016/j. annemergmed.2011.11.036

15. van der Linden MC, van den Brand CL, van der Linden N, Rambach $\mathrm{AHJH}$, Brumsen C. Rate, characteristics, and factors associated with high emergency department utilization. Int J Emerg Med. 2014;7 (1):9. doi:10.1186/1865-1380-7-9

16. Riggs JE, Davis SM, Hobbs GR, Paulson DJ, Chinnis AS, Heilman PL. Association between early returns and frequent ED visits at a rural academic medical center. Am J Emerg Med. 2003;21(1):30-31. doi:10.1053/ajem.2003.50017

17. Jelinek GA, Jiwa M, Gibson NP, Lynch A-M. Frequent attenders at emergency departments: a linked-data population study of adult patients. Med J Aust. 2008;189(10):552. doi:10.5694/j.1326-5377.20 08.tb02177.x

18. Gibson NP, Jelinek GA, Jiwa M, Lynch A. Paediatric frequent attenders at emergency departments: a linked-data population study. $J$ Paediatr Child Health. 2010;46(12):723-728. doi:10.1111/j.14401754.2010.01829.x

19. Capp R, Rosenthal MS, Desai MM, et al. Characteristics of Medicaid enrollees with frequent ED use. Am J Emerg Med. 2013;31(9):13331337. doi:10.1016/j.ajem.2013.05.050

20. Doran KM, Raven MC, Rosenheck RA. What drives frequent emergency department use in an integrated health system? National data from the Veterans Health Administration. Ann Emerg Med. 2013;62 (2):151-159. doi:10.1016/j.annemergmed.2013.02.016

21. Bullard MJ, Unger B, Spence J, Grafstein E; Group CNW. Revisions to the Canadian emergency department triage and acuity scale (CTAS) adult guidelines. Can J Emerg Med. 2008;10(2):136-142. doi:10.1017/S1481803500009854

22. Salway RJ, Valenzuela R, Shoenberger JM, Mallon WK, Viccellio A. Emergency department (ed) overcrowding: evidence-based answers to frequently asked questions. Rev Médica Clínica Las Condes. 2017;28(2):213-219. doi:10.1016/j.rmclc.2017.04.008
23. Al-Arifi M, Abu-Hashem H, Al-Meziny M, Said R, Aljadhey H. Emergency department visits and admissions due to drug related problems at Riyadh military hospital (RMH), Saudi Arabia. Saudi Pharm J SPJ off Publ Saudi Pharm Soc. 2014;22(1):17-25. doi:10.1016/j.jsps.2013.01.001

24. Alhabdan N, Alhusain F, Alharbi A, Alsadhan M, Hakami M, Masuadi E. Exploring emergency department visits: factors influencing individuals' decisions, knowledge of triage systems and waiting times, and experiences during visits to a tertiary hospital in Saudi Arabia. Int J Emerg Med. 2019;12(1):35. doi:10.1186/s12245-0190254-7

25. Al-Otmy SS, Abduljabbar AZ, Al-Raddadi RM, Farahat F. Factors associated with non-urgent visits to the emergency department in a tertiary care centre, western Saudi Arabia: cross-sectional study. BMJ Open. 2020;10(10):e035951. doi:10.1136/bmjopen-2019-035951

26. Zakria I, Ala'ddin A, Alnatour M, Bashir M. Myth versus reality emergency department revisits within 72 hours, at a tertiary care hospital In Saudi Arabia. 2020. doi:10.21203/rs.3.rs-30756/v1

27. Ahmed A, AlBuraikan D, ALMuqbil B, AlJohi W, Alanazi W, AlRasheed B. Readmissions and emergency department visits after bariatric surgery at Saudi Arabian Hospital: the rates, reasons, and risk factors. Obes Facts. 2017;10(5):432-443. doi:10.1159/000456667

28. Ahmed AE, Alaskar AS, McClish DK, et al. Saudi SCD patients' symptoms and quality of life relative to the number of $\mathrm{ED}$ visits. BMC Emerg Med. 2016;16(1):30. doi:10.1186/s12873-016-0096-z

29. Shaw JE, Sicree RA, Zimmet PZ. Global estimates of the prevalence of diabetes for 2010 and 2030. Diabetes Res Clin Pract. 2010;87 (1):4-14. doi:10.1016/j.diabres.2009.10.007

30. Bieler G, Paroz S, Faouzi M, et al. Social and medical vulnerability factors of emergency department frequent users in a universal health insurance system. Acad Emerg Med. 2012;19(1):63-68. doi:10.1111/ j.1553-2712.2011.01246.x

31. Hooker EA, Mallow PJ, Oglesby MM. Characteristics and trends of emergency department visits in the United States (2010-2014). $J$ Emerg Med. 2019;56(3):344-351. doi:10.1016/j.jemermed.2018. 12.025

32. Liu SW, Nagurney JT, Chang Y, Parry BA, Smulowitz P, Atlas SJ. Frequent ED users: are most visits for mental health, alcohol, and drug-related complaints? Am J Emerg Med. 2013;31(10):1512-1515. doi:10.1016/j.ajem.2013.08.006

33. Vinton DT, Capp R, Rooks SP, Abbott JT, Ginde AA. Frequent users of US emergency departments: characteristics and opportunities for intervention. Emerg Med J. 2014; emermed-2013-202407.

34. LaCalle EJ, Rabin EJ, Genes NG. High-frequency users of emergency department care. J Emerg Med. 2013;44(6):1167-1173. doi:10.1016/j.jemermed.2012.11.042

35. Kirby SE, Dennis SM, Jayasinghe UW, Harris MF. Patient related factors in frequent readmissions: the influence of condition, access to services and patient choice. BMC Health Serv Res. 2010;10(1):216. doi:10.1186/1472-6963-10-216

36. Markham D, Graudins A. Characteristics of frequent emergency department presenters to an Australian emergency medicine network. BMC Emerg Med. 2011;11(1):21. doi:10.1186/1471-227X-11-21

37. Chukmaitov AS, Tang A, Carretta HJ, Menachemi N, Brooks RG. Characteristics of all, occasional, and frequent emergency department visits due to ambulatory care-sensitive conditions in Florida. $J$ Ambul Care Manag. 2012;35(2):149-158. doi:10.1097/JAC.0b013e31824 $4 \mathrm{~d} 222$

38. Salazar A, Bardés I, Juan A, Olona N, Sabido M, Corbella X. High mortality rates from medical problems of frequent emergency department users at a university hospital tertiary care centre. Eur J Emerg Med. 2005;12(1):2-5. doi:10.1097/00063110-200502000-00002

39. Moradi-Lakeh M, El Bcheraoui C, Daoud F, et al. Prevalence of asthma in Saudi adults: findings from a national household survey, 2013. BMC Pulm Med. 2015;15(1):77. doi:10.1186/s12890-0150080-5 
40. Elkum NB, Barrett C, Al-Omran H. Canadian Emergency DepartmentTriage and Acuity Scale: implementation in a tertiary care center in Saudi Arabia. BMC Emerg Med. 2011;11(1):3. doi:10. 1186/1471-227X-11-3

41. Fernandes CMB, McLeod S, Krause J, et al. Reliability of the Canadian Triage and Acuity Scale: interrater and intrarater agreement from a community and an academic emergency department. Can J Emerg Med. 2013;15(4):227-232. doi:10.2310/8000.2013.130943

42. Genell Andrén K, Ahmed AE, Alaskar AS, McClish DK. A study of the relationship between social network, perceived ill health and utilization of emergency care. A case-control study. Scand J Soc Med. 1988;16(2):87-93. doi:10.1177/140349488801600205
43. Rask KJ, Williams MV, McNagny SE, Parker RM, Baker DW. Ambulatory health care use by patients in a public hospital emergency department. J Gen Intern Med. 1998;13(9):614-620. doi:10. 1046/j.1525-1497.1998.00184.x

44. Pope D, Fernandes CMB, Bouthillette F, Etherington J. Frequent users of the emergency department: a program to improve care and reduce visits. CMAJ. 2000;162(7):1017-1020.

\section{Publish your work in this journal}

The International Journal of General Medicine is an international, peer-reviewed open-access journal that focuses on general and internal medicine, pathogenesis, epidemiology, diagnosis, monitoring and treatment protocols. The journal is characterized by the rapid reporting of reviews, original research and clinical studies across all disease areas. The manuscript management system is completely online and includes a very quick and fair peer-review system, which is all easy to use. Visit http://www.dovepress.com/ testimonials.php to read real quotes from published authors.

Submit your manuscript here: https://www.dovepress.com/international-journal-of-general-medicine-journal 